\title{
NON-TRAUMATIC PROGRESSIVE PARALYSIS OF THE POSTERIOR INTEROSSEOUS NERVE
}

\author{
R. C. Mulholland, London, England \\ From the Orthopaedic Department, St Bartholomew's Hospital, London
}

Isolated lesions of the posterior interosseous nerve of the forearm are uncommon. Most follow injury to the elbow region (Roussy and Branche 1917, Mowell 1921, Naylor 1942). The clinical features of a long-standing lesion of the nerve are wasting of the posterior forearm muscles (excepting brachio-radialis and extensor carpi radialis longus), diminution or loss of active extension of the fingers at the metacarpo-phalangeal joints, and weakness or paresis of the abductor pollicis longus muscle. There is no wrist drop as the nerve supply to the extensor carpi radialis longus is intact, but on extension the wrist tends to drift into radial deviation because of the paralysis of extensor carpi ulnaris muscle.

A number of cases have now been reported of an almost painless progressive paralysis of this nerve, usually unexplained.

\section{ANATOMY}

The radial nerve terminates a few centimetres above the lateral condyle of the humerus, dividing into the posterior interosseous nerve and the lateral cutaneous nerve of the forearm. Before this division, branches of supply are given to the brachialis, brachio-radialis and extensor carpi radialis longus muscles. The posterior interosseous nerve descends in the cleft between the brachialis and brachio-radialis, passes under the extensor carpi radialis longus and brevis, supplying the latter muscle, and then pierces the supinator. It curves around the radius in the substance of the supinator and usually, at the lower border, divides into two branches, one of which supplies the medial extensor muscles-extensor carpi ulnaris, extensor digitorum and extensor digiti minimi-and the other supplies the abductor pollicis longus and brevis and the extensor indicis muscles. Occasionally some of the fibres of the latter branch pass entirely superficial to the supinator or become superficial earlier in their course (Luschka and Krause 1927). The nerve is well overlapped by muscle throughout its course, and over the extensor muscles there is a tough unyielding aponeurosis.

\section{PREVIOUS REPORTS}

I have been able to find twenty reported cases of paralysis of the posterior interosseous nerve apparently unrelated to injury. In only seven of these has exploration been carried out. Agnew (1863) explored the forearm of a woman who had both flexor and extensor weakness. An exquisitely tender tumour " about the size of a hickory nut " was palpable anteriorly close to the biceps tendon. Exploration of this swelling showed it to be a solid lesion containing fibrous and connective tissue, lying between the tubercle of the radius and the biceps tendon. It was apparently compressing both the posterior interosseous and the median nerves. Its removal was followed by recovery. Weinberger (1939) quoted Nancrede's observation of a patient with a bursa between the common extensor muscles and the extensor carpi ulnaris which compressed the posterior interosseous nerve. In 1905 Guillain and Courtellemont reported a case in an orchestral conductor, ascribing the trouble to the repeated movements of pronation and supination when conducting. Jumentie (1921) reported a case associated with a swelling on the dorsum of the wrist, extending up the forearm. Grigoresco and Iordanesco (1931) reported paralysis of the nerve after minor arm strain made worse by sleeping with the head on the forearm. In none of these patients was exploration carried out.

In 1934 Woltman and Learmonth reported five personal cases, with exploration in only one. In that case the only abnormality found was that the nerve lay entirely superficial to the supinator muscle, which it grooved. It was supposed that it was compressed between the 
extensor muscles and the supinator. A strip of aponeurosis overlying the nerve was excised and the superficial muscles were not repaired. Despite this decompression the nerve did not recover its function.

In 1936 Hobhouse and Heald reported a case in a barman who was a total abstainer. This began with weakness of the three ulnar fingers of his left hand, associated with a painful elbow. The pain improved but the paresis extended, and after a month there was complete paresis of all muscles supplied by the posterior interosseous nerve. There was no weakness of the supinator muscle. Exploration was not carried out. In 1937 Silverstein reported a patient with a history of six years gradual progression of paresis, which had started while he was playing a violin several hours a day. Again apparently supination was spared. In 1939 Weinberger reported two cases, the first occurring in a dairyman, seemingly after a wrench of his arm, and the second in a corsetière, who had a small mobile tender nodule over the insertion of the biceps tendon. In the dairyman pressure in this area also produced pain, but no swelling was detected. Weinberger ascribed the lesion to a chronic bursitis of the bicipital bursa. In neither patient was the nerve explored.

In 1947 Otenasek reported a single case. The patient was a man of twenty-one with weakness of the left hand. Weakness had appeared first in the thumb, and spread to all the fingers. Supination was weak. Exploration of the nerve at the elbow revealed a swelling in the nerve at its beginning. Histologically this was connective tissue, which was very cellular and contained many collagen fibres. It was considered to be either a scarred nerve or a fibrous tumour of nerve.

Richmond (1953), Hustead, Mulder and MacCarty (1958) and Cappellini (1958) all reported cases in which lipomata were found. The position of the lipoma varied and this affected the ease with which it was detected. In one of Hustead's cases it was easily felt, being attached to the capsule of the superior radio-ulnar joint. In another it was difficult to detect as it lay deep to the extensor muscles. In Richmond's case the lipoma lay deep to the terminal branches of the radial nerve and was easily felt.

Kruse (1958) reported a unique case in that spontaneous recovery occurred. There was no history of injury. The paresis appeared after swimming and was associated with a very painful elbow and tenderness in the region of the bicipital bursa. It persisted for six months unchanged and then completely recovered during the next three months.

\section{CASE REPORT}

A right-handed man of twenty-seven had suffered for ten years from weakness of extension of the left thumb and fingers. At the age of seven he was given an injection into the left upper arm which afterwards swelled and he developed wrist drop. The arm was rested in plaster and he was given local heat to his wrist. Recovery occurred in six months. Ten years later he noticed that he could not extend his left index finger, and during the following year the other fingers were also affected. There was no wrist drop and supination was not affected. He was rejected for National Service because of the paresis which, because it came on just before his call-up, was believed to be hysterical. He continued his occupation of a veneer preparer. In this work his left hand was used to steady the already fixed veneer while the right hand made rapid to-and-fro movements to flatten and match the next piece.

Examination showed marked wasting of the extensors of thumb and fingers, but powerful contraction of brachio-radialis and extensor carpi radialis longus muscle was present. A tender spot was felt 8 centimetres below the elbow on the back of the forearm, but no swelling was detected.

Operation-Through a postero-lateral approach the radial nerve was exposed and its posterior branch followed. At the lower border of the supinator two small nodules were seen on the nerve, and beyond them the nerve appeared thin and atrophic. The nodules and part of the adjacent nerve were excised. 
Histology-The excised specimen consisted of a piece of grey tissue measuring $0.4 \times 0.3 \times 0.2$ centimetre. The nerve showed interstitial fibrosis which distorted the normal architecture of the nerve bundles and surrounded the individual nerve fibres. In some areas the fibrous tissue showed myxoid change. No specific etiology was suggested by these appearances, but they would be consistent with old trauma or vascular damage.

Progress-After operation there was no change in the extent of his paresis and he is to have tendon transplantation.

Comment-Paralysis of the posterior interosseous nerve does not cause severe disability, but enough to make the patient likely to seek medical advice. Although twenty cases have now been described, the cause remains speculative in most. Before making the diagnosis of posterior interosseous nerve palsy, lead palsy and hysteria must be excluded. In early lead palsy the paresis may be restricted to the extensor digitorum communis, sparing the brachio-

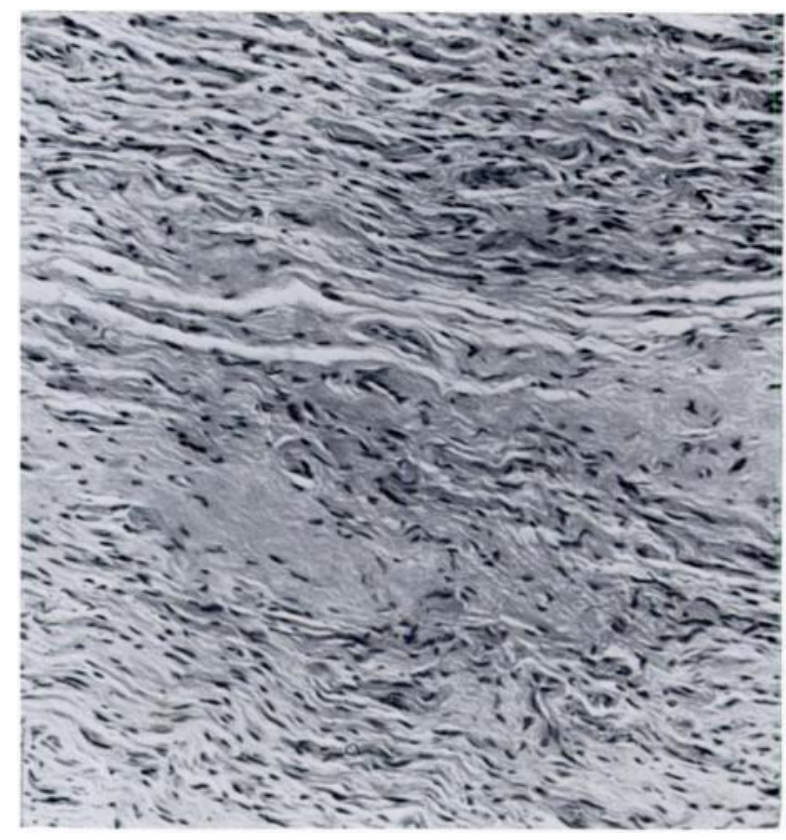

Fig. 1

Section of excised tissue showing interstitial fibrosis and areas of myxoid change. (Haematoxylin and eosin, $\times 115$.) radialis muscle; it thus mimicks two important features of a posterior interosseous nerve lesion. However, the abductor pollicis longus is also spared, which is not the case in the true lesion (Hunter 1962). Lead palsy progresses to involve both the wrist extensors, causing complete wrist drop, and there will be other evidence of plumbism. Hysterical wrist drop can be distinguished because the patient fails to extend the terminal phalanges in any position of the metacarpo-phalangeal joints; that is, the extensor action of the intrinsic muscles is also absent.

Among the seven cases in which exploration was carried out, no less than five were due to pressure from a space-occupying lesion, four lipomata and one probable fibroma. In these patients the history showed no typical features and, surprisingly, the onset of the paresis was often sudden and progression was rapid. The lipomata were all palpable, and their relative translucency on radiographs was used to confirm the clinical impression in two cases (Richmond 1953. Hustead et al. 1958). These findings suggest that some of the reported cases in which the forearm was not explored were due to pressure from an undetected tumour. Ganglia are not uncommonly a cause of peripheral nerve paresis (Brooks 1952, Seddon 1952), yet none appears to have been incriminated at this site. The small mobile nodule described by Weinberger in one of his cases may well have been a ganglion arising from the superior radio-ulnar joint.

It was suggested by Woltman and Learmonth (1934) that the superficial course of the posterior interosseous nerve over the supinator rendered it liable to compression. More recently Capener $(1960,1964)$ expressed a similar opinion, having seen several patients with weakness of the forearm muscles relieved by decompression of the nerve. As the posterior interosseous nerve gives some branches of supply to the supinator before entering it, a compressive lesion here would not fully paralyse this muscle. This might explain why weakness of supination was seldom present.

VOL. 48 B, NO. 4, NOVEMBER 1966 
Weinberger (1939) postulated bicipital bursitis as a cause in some instances because of the close relationship between the bursa and the posterior interosseous nerve before it enters the supinator. However, if this were so, weakness of supination would usually be present. An acute bursitis affecting the insertion of the biceps muscle would be associated with painful flexion. This was present in only two patients, one of whom was atypical in that recovery occurred. The existence of associated pain does not indicate an inflammatory cause, as it was present with a benign lipoma (Richmond 1953).

Since this lesion was first described in an orchestral conductor (Guillain and Courtellemont 1905), occupation has ranked high on the list of possible causes. It was postulated that repeated and alternating pronation-supination movement caused injury to the nerve. However, the occupations quoted in support of this possibility-violinist, pianist and corsetière do not require this movement unduly. In those industries in which work does involve much of this movement, for instance the net making industry in Northern Ireland, this particular lesion is not met with (Smiley 1951). Marinacci (1960) described eleven differing pressure neuropathies related to various occupations; not only has he not met one involving the posterior interosseous nerve, but these occupational neuropathies recovered when the occupation was altered. The non-recurrence of any one occupation (except housewife) in the reported cases makes an occupational cause unlikely.

The histological features in our own case and that reported by Otenasek (1947) are similar. Otenasek interpreted these as representing an atypical fibroma or alternatively a scarred nerve. He rejected the latter possibility in view of the absence of a history of injury. However, the histology in our case showed the collagen and fibrous tissue arranged in a linear fashion parallel to the nerve and not at random as in a fibroma. We suggest that both these lesions are similar and possibly due to unremembered injury interfering with blood supply and leading to a slow replacement fibrosis of the nerve. Paresis of the ulnar nerve is a not uncommon late sequel of certain elbow injuries and a patient of the late W. D. Coltart showed a similar process in the posterior interosseous nerve. She was a girl who at the age of three had injured her elbow. Six months after the accident it was first noticed that she had wrist drop. When she was seen at the age of six radiography showed a postero-lateral dislocation of the head of the radius. Clinical and electromyographic tests confirmed a complete posterior interosseous nerve lesion. This case resembles that reported by Adams and Rizzoli in 1959, of a man of fifty, who when aged three had dislocated his elbow and was left with a dislocated head of radius. His posterior interosseous nerve lesion did not appear for forty-seven years, but was cured when the radial head was excised.

\section{TREATMENT}

In patients with unexplained posterior interosseous nerve lesions exploration of the nerve is obviously justifiable. If no remediable cause is found the disability can be helped greatly by appropriate tendon transfers. The deficit is the loss of extensor digitorum communis and the abductor pollicis longus. The synergistic muscles available for transfer are the wrist flexors (including palmaris longus if present). There are a number of variations in how these motor tendons are used. Zachary (1946) assessed several of these. He recalled the well established importance of leaving one carpal flexor undisturbed: transfer of both radial and ulnar carpal flexors gives poor results if palmaris longus is absent or weak. Usually the tendon of flexor carpi ulnaris is transferred into all the extensor tendons of the fingers and to extensor pollicis longus. White (1960) points out that fixation to the extensor digiti minimi should be considerably looser than that to the extensor communis, because otherwise the little finger will tend to overextend. The palmaris longus if present is transferred to the extensor pollicis brevis and abductor pollicis longus; if the palmaris longus is absent the sublimis tendon of the ring finger may be used. A good result may be achieved even if abductor pollicis is not replaced. Tendon junctures should be placed proximal to the transverse carpal ligament. 
SUMMARY

1. A case of spontaneous posterior interosseous paresis is reported. It is suggested that the cause was replacement fibrosis secondary to local ischaemic damage from unremembered minor trauma.

2. In a patient with a posterior interosseous nerve paresis examination may reveal a spaceoccupying lesion near the elbow along the course of the nerve thus compressing it. Recovery may be expected after its removal. Consequently the nerve should be explored before resort to tendon transfers.

I wish to thank Mr J. N. Aston for his help and advice in the preparation of this paper, and for permitting me to publish the case. My thanks are due also to Dr A. G. Stansfeld for the histology and much helpful advice.

\section{REFERENCES}

Adams, J. R., and Rizzoli, H. V. (1959): Tardy Radial and Ulnar Nerve Palsy. Journal of Neurosurgery, 16, 342. Agnew, D. H. (1863): Bursal Tumour Producing Loss of Power of Forearm. American Journal of Medical Science, 46, 404.

Brooks, D. M. (1952): Nerve Compression by Simple Ganglia. Journal of Bone and Joint Surgery, 34-B, 391. CAPEner, N. (1960): Tennis Elbow and Posterior Interosseous Nerve. British Medical Journal, ii, 130.

CAPener, N. (1964): Posterior Interosseous Nerve Lesions. Journal of Bone and Joint Surgery, 46-B, 361.

CAPpellini, O. (1958): Su un caso di paralisi dissociata di radiale da lipoma intermuscolare. Chirurgia degli Organi di Movimento (Bologna), 45, 338.

Grigoresco, D., and Iordanesco, C. (1931): Un cas rare de paralysie partielle du nerf radial. Revue Neurologique, 38, 102.

Guillain, G., and Courtellemont (1905): L'action du muscle court supinateur dans la paralysie du nerf radial. Presse Médicale, 13, 50.

Hobhouse, N., and Heald, C. B. (1936): A Case of Posterior Interosseous Paralysis. British Medical Journal, i, 841.

Hunter, D. (1962): The Diseases of Occupation. Third edition. London: The English Universities Press Ltd.

Hustead, A., Mulder, D., and MacCarty, C. (1958): Nontraumatic Progressive Paralysis of the Deep Radial (Posterior Interosseous) Nerve. Archives of Neurology and Psychiatry, 79, 269.

JumentIÉ, J. (1921): Fausse griffe cubitale par lésion dissociée du nerf radial. Revue Neurologique, 28, 756.

KruSE, F., Jun. (1958): Paralysis of the Dorsal Interosseous Nerve not due to Direct Trauma. A Case showing Spontaneous Recovery. Neurology (Minneapolis), 8, 307.

LuschKa and KraUSE (1927): Quoted by Hovelacque, A. Anatomie des nerfs craniens et rachidiens et du système grand sympathique. Paris: G. Doin et Cie.

MarinaCCI, A. A. (1960): Some Unusual Causes of Pressure Neuropathies. Bulletin of the Los Angeles Neurological Society, 25, 223.

Mowell, J. W. (1921): Posterior Interosseous Nerve Injury. International Clinics. Thirty-first series, 2, 188.

NANCREDE, C. B. (1882): Bursae of Elbow and Vicinity. In International Encyclopedia of Surgery, Vol. $2,711$. Edited by J. Ashhurst. New York: W. Wood \& Co.

NAYLOR, A. (1942): Monteggia Fractures. British Journal of Surgery, 29, 323.

Otenasek, F. J. (1947): Progressive Paralysis of the Nervus Interosseous Dorsalis. Pathological Findings in One Case. Bulletin of the Johns Hopkins Hospital, 81, 163.

Richmond, D. A. (1953): Lipoma Causing a Posterior Interosseous Nerve Lesion. Journal of Bone and Joint Surgery, 35-B, 83.

Roussy, G., and BRANCHE, J. (1917): Deux cas de paralysies dissociées de la branche postérieure du radial, à type de pseudo-griffe cubitale. Revue Neurologique, 24, 312.

Seddon, H. J. (1952): Carpal Ganglion as a Cause of Paralysis of the Deep Branch of the Ulnar Nerve. Journal of Bone and Joint Surgery, 34-B, 386.

Silverstein, A. (1937): Progressive Paralysis of the Dorsal Interosseous Nerve. Archives of Neurology and Psychiatry, 38, 885.

Smiley, J. A. (1951): The Hazards of Rope Making. British Journal of Industrial Medicine, 8, 265.

Weinberger, L. M. (1939): Non-traumatic Paralysis of the Dorsal Interosseous Nerve. Surgery, Gynecology and Obstetrics, 69, 358.

WhITE, W. L. (1960): Restoration of Function and Balance of the Wrist and Hand by Tendon Transfers. Surgical Clinics of North America, 40, 427.

Woltman, H. W., and Learmonth, J. R. (1934): Progressive Paralysis of the Nervus Interosseous Dorsalis. Brain, 57, 25.

ZACHARY, R. B. (1946): Tendon Transplantation for Radial Paralysis. British Journal of Surgery, 33, 358.

VOL. 48 B, NO. 4, NOVEMBER 1966 